\title{
Utilização das tecnologias de informação e comunicação pelos núcleos ampliados de saúde da família
}

\author{
Use of information and communication technologies by Family Health Extended Centers
}

Uso de tecnologías de información y comunicación por los Núcleos Ampliados de Salud de la Familia

\section{Carine VendruscoloI, Daniela Savi Geremia ${ }^{\mathrm{II}}$, Edlamar Kátia Adamy ${ }^{\mathrm{III}}$, Lara Vandresen ${ }^{\mathrm{IV}}$, Lucimare Ferraz ${ }^{\mathrm{V}}$}

\begin{abstract}
Resumo: Objetivo: analisar a utilização das tecnologias de informação e comunicação pelos Núcleos Ampliados de Saúde da Família e refletir sobre seu potencial para o cuidado. Método: estudo de métodos mistos, com amostra quantitativa de 359 profissionais dos Núcleos, em 149 municípios, mediante aplicação de Survey, e etapa qualitativa por grupos focais com cinco equipes, 43 nasfianos. Dados coletados entre maio e novembro de 2017 e tratados conforme abordagem estatística descritiva e análise temática. Resultados: dentre as tecnologias, destacam-se os prontuários eletrônicos e o diálogo entre os profissionais das equipes de Saúde da Família com os nasfianos. O agente comunitário é acionado com frequência de diálogo, porém há reduzida participação de lideranças e conselheiros de saúde. Conclusão: apesar dos avanços, a utilização das tecnologias ainda não atinge todo o seu potencial para o cuidado, sendo necessário estimular o diálogo entre equipes, a utilização do mapa do território e a participação social.
\end{abstract}

Descritores: Atenção primária à saúde; Desenvolvimento tecnológico; Acesso aos serviços de saúde; Administração de serviços de saúde; Enfermagem

Abstract: Objective: To analyze the use of information and communication technologies by Family Health Extended Centers and to reflect on their potential for care. Method: study of mixed methods, with a quantitative sample of 359 professionals from the nuclei, in 149 municipalities, with survey application, and qualitative step

\footnotetext{
${ }^{\text {I }}$ Doutora em Enfermagem, professora adjunta do Departamento de Enfermagem e vice coordenadora do Programa de Pós-graduação em Enfermagem da Universidade do Estado de Santa Catarina (UDESC). Chapecó, Santa Catarina, Brasil. E-mail: carine.vendruscolo@udesc.br Orcid: http://orcid.org/0000-0002-5163-4789

II Doutora em Enfermagem, professora da Universidade Federal da Fronteira Sul (UFFS). Chapecó, Santa Catarina, Brasil. E-mail: daniela.savi.geremia@gmail.com Orcid: http://orcid.org/0000-0003-2259-7429

III Doutora em Enfermagem, professora adjunta do Departamento de Enfermagem, Graduação e Programa de Pós-graduação em Enfermagem da Universidade do Estado de Santa Catarina (UDESC). Chapecó, Santa Catarina, Brasil. Email: edlamar.adamy@udesc.br Orcid: https://orcid.org/0000-0002-8490-0334

IV Doutoranda pelo Programa de Pós-graduação em Enfermagem da Universidade Federal de Santa Catarina (UFSC). Florianópolis, Santa Catarina, Brasil. E- mail: laravandresen@hotmail.com Orcid: http://orcid.org/0000-0002-1389-7932

${ }^{v}$ Doutora em Enfermagem, professora adjunta do Departamento de Enfermagem, Graduação e Programa de Pós-graduação em Enfermagem da Universidade do Estado de Santa Catarina (UDESC). Chapecó, Santa Catarina, Brasil. E-mail: lucimare.ferraz@udesc.br Orcid: http://orcid.org/0000-0002-2487-8614
} 
comprising focus groups with five teams, 43 from the nuclei. Data was collected between May and November 2017 and treated according to descriptive statistical approach and thematic analysis. Results: Among technologies, electronic medical records and dialogue between professionals of the Family Health teams and the nuclei are highlighted. Dialogue with community agents is frequent, but there is little participation of health leaders and counselors. Conclusion: Despite the advances, the use of technologies still does not reach its full potential for care, and one must stimulate dialogue among teams, the use of territory map and social participation.

Descriptors: Primary Health Care; Technological development; Access to health services; Health services administration; Nursing

Resumen: Objetivo: analizar uso de tecnologías de información y comunicación de Núcleos Ampliados de Salud de la Familia y reflexionar sobre su potencial de atención. Método: estudio de métodos mixtos, muestra cuantitativa de 359 profesionales de los Núcleos en 149 municipios, mediante aplicación de encuesta, y etapa cualitativa con grupos focales en cinco equipos, 43 del Núcleo. Datos recopilados entre mayo y noviembre de 2017 y tratados según enfoque estadístico descriptivo y análisis temático. Resultados: Entre las tecnologías, destacamos registros médicos electrónicos y diálogo entre profesionales de los equipos de Salud de la Familia y los del Núcleo. Diálogo con agentes comunitarios es frecuente, pero hay poca participación de líderes y consejeros de salud. Conclusión: a pesar de los avances, el uso de tecnologías aún no alcanza su máximo potencial para la atención; es necesario estimular el diálogo entre equipos, el uso del mapa del territorio y la participación social.

Descriptores: Atención primaria de salud; Desarrollo tecnológico; Acceso a servicios de salud; Administración de servicios de salud; Enfermería

\section{Introdução}

As tecnologias de informação e comunicação (TICs) em saúde são ferramentas utilizadas para a melhoria dos processos e armazenamento das informações - por exemplo, os sistemas de computação. Na Atenção Primária à Saúde (APS), recursos como prontuários eletrônicos vêm ganhando destaque e investimento em nível internacional. Este tipo de TIC interfere diretamente na qualidade da interface com o usuário e integração com sistemas externos, impactando na eficiência da comunicação e coordenação do cuidado na prática clínica, com resultados para os pacientes crônicos. ${ }^{1}$ Informações integradas e em tempo oportuno são ferramentas que subsidiam os processos de tomada de decisão e qualificam a atenção para além do foco assistencialista; nessa direção, o diálogo entre equipes também opera como dispositivo tecnológico. ${ }^{2}$ 
O processo de incorporação das TICs, sobretudo na APS, tem avançado significativamente, e sua disponibilidade e uso são fundamentais para promover cuidados com maior segurança. O governo federal, por meio do Ministério da Saúde (MS), tem feito esforços importantes para que as equipes que atuam na APS utilizem os sistemas de informação para a qualificação dos processos de trabalho gerenciais e assistenciais, pois eles proporcionam novas interpretações de eventos e fenômenos, além de auxiliarem na elaboração de planejamentos e autonomia para a formulação e implementação de políticas públicas e de cuidado integral e longitudinal aos usuários do Sistema Único de Saúde (SUS). ${ }^{3}$

A relação entre inovação e desenvolvimento permite a inserção de novas tecnologias no setor saúde. Embora ainda seja precária, na APS a articulação entre serviços e ações para o cuidado integral pode ser potencializada pela utilização das TICs, assim como a continuidade informacional entre Atenção Primária e Secundária, por meio do prontuário eletrônico, por exemplo. ${ }^{4}$ Entretanto, a tecnologia, sob diversas modalidades, tem sido incorporada gradativamente à assistência e à gestão em saúde, inclusive à distância, tendo como uma das principais preocupações a redução de custos. ${ }^{5}$

Os Núcleos Ampliados de Saúde da Família e Atenção Básica (Nasf-AB) configuram uma política para estimular a consolidação da APS no Brasil, mediante a composição de equipes multiprofissionais que operam na perspectiva do apoio matricial junto às equipes de Saúde da Família (eSF), com vistas a fortalecê-las e ampliar sua resolubilidade. A diversidade de profissões passíveis de integração às equipes generalistas (de Saúde da Família) confere ao Nasf a possibilidade de desempenhar atividades interdisciplinares, considerando os conhecimentos dos núcleos específicos de cada profissão e o campo de saberes dos cuidados em saúde comuns a todas as profissões. ${ }^{6-7}$

Ao considerar-se o modelo que opera na APS, o desafio para a atuação das equipes dos Nasf-AB é ainda maior do que o das eSF, dadas as características especiais das atividades 
Utilização das tecnologias de informação e comunicação pelos núcleos ampliados de saúde... | 4

desempenhadas por essa equipe de apoio e suporte, uma vez que ela é altamente dependente da integração do sistema com outras atividades e dispositivos sociais. Entre os desafios, destacamse: a falta de estrutura, o pouco uso de instrumentos organizacionais de trabalho, o frágil vínculo empregatício e a rotatividade de profissionais. ${ }^{7}$

A organização de cada Unidade Básica de Saúde e o processo de trabalho dos profissionais da eSF e Nasf são bastante heterogêneos, alternam demandas e situações diversas, necessitando de informações de alta confiabilidade para a comunicação e desenvolvimento das atividades planejadas, as quais podem ser intensificadas por meio das TICs. O trabalho das equipes de Nasf- $\mathrm{AB}$, com certa frequência, acaba sendo imediato para atender às demandas, com o desenvolvimento de ações e intervenções com pouco ou nenhum planejamento e monitoramento das situações individuais e coletivas. ${ }^{8}$

As mudanças tecnológicas e as transformações recorrentes relacionadas às TICs estão diretamente implicadas nos processos de trabalho e impactam as formas de gerenciamento e organização dos serviços de saúde. Entre os dilemas observados nos cotidianos das eSF e dos Nasf- $A B$, nota-se a subutilização da potencialidade das novas tecnologias incorporadas, que mesmo com o esforço do MS e secretarias de Saúde, fica à mercê da possibilidade de uso e resolução de problemas no setor saúde. Percebe-se a dificuldade dos profissionais das equipes do Nasf-AB e demais profissionais da eSF para dispor e utilizar informações, bem como outros dispositivos sociais de apoio aos serviços de saúde e à própria rede de atenção. Nesse sentido, faz-se necessário incluir ações e aparatos tecnológicos que promovam o conhecimento voltado a prevenção, promoção e reabilitação da saúde na APS. Além disso, que se promova o fortalecimento de uma cultura que valorize o uso da informação para subsidiar o processo de trabalho. ${ }^{4}$

Com base no contexto apresentado, emergem as questões: quais as fontes de informação utilizadas pelas equipes dos Nasf-AB que se caracterizam como TIC? Como elas são utilizadas 
5 | Vendruscolo C, Geremia DS, Adamy EK, Vandresen L, Ferraz L

para a qualificação das relações e melhorias nos processos de trabalho das equipes? A partir dessas indagações, o estudo tem como objetivo analisar a utilização das TIC pelos Nasf-AB e refletir sobre seu potencial para o cuidado.

\section{Método}

Pesquisa multicêntrica, de métodos mistos, em cuja produção e análise dos dados foi utilizada abordagem quanti-qualitativa, estratégia exploratória e sequencial. A realização de um estudo de métodos mistos não implica a condução de pesquisas separadas que abordam uma questão específica, mas a inclusão de métodos diferentes para responder à questão, mediante informações complementares. ${ }^{9}$ O estudo foi desenvolvido por pesquisadores de cinco universidades de SC (duas públicas, duas comunitárias e uma privada), com participação da Secretaria de Estado da Saúde de Santa Catarina (SC).

Os participantes foram profissionais das equipes do Nasf-AB de SC. Para a etapa quantitativa, foi realizado cálculo amostral, que definiu o quantitativo de participantes dentre os 1.312 profissionais do Nasf-AB atuantes no Estado. Esta etapa ocorreu mediante aplicação de survey, encaminhado via correio eletrônico, para todos os profissionais nasfianos (que atuam no Nasf-AB). Como critério de inclusão, era necessário estar formalmente atuando no Nasf-AB há, pelo menos, um ano. Foram excluídos os profissionais afastados por qualquer motivo durante o período.

A amostra da etapa quantitativa foi composta por 359 profissionais do Nasf-AB, atuantes em 149 municípios do Estado de SC, distribuídos nas nove Macrorregiões de Saúde. Para participar, ao receber o e-mail convite, era necessário clicar em “aceitar”, o que dava acesso ao Termo de Consentimento Livre Esclarecido (TCLE) e liberava o survey. Os profissionais responderam ao questionário na ferramenta survey, com 19 questões fechadas do tipo Likert e abertas, incluindo dados como características do território, população assistida, principais 
Utilização das tecnologias de informação e comunicação pelos núcleos ampliados de saúde... | 6

agravos, entre outros; além de questionamentos sobre o processo de trabalho e demandas da atuação das equipes. O instrumento obteve elevado coeficiente alfa de Cronbach $(\alpha=0,819)$ e passou por um teste, para avaliação da consistência e linguagem. Essa etapa ocorreu entre maio e junho de 2017.

O procedimento analítico das informações quantitativas ocorreu mediante codificação e digitação dos dados no programa Exce跑, sendo tabulados e analisados com auxílio do Statistical Package for the Social Sciences (SPSS) ${ }^{\circledR}$, versão 21.0. As variáveis desta natureza passaram por descrição, a partir de medidas de tendência central e dispersão: média, desvio padrão, mediana e valor mínimo e máximo observados, estimativa por intervalo de confiança para a média populacional com base no número de respostas válidas, considerando nível de significância menor que $5 \%(\mathrm{p}<0,05)$, enquanto o intervalo de confiança foi igual ou maior que $95 \%$. Descreveram-se as variáveis categóricas por meio de frequências absolutas e proporções, sendo apresentadas neste estudo aquelas que têm relação com o tema proposto.

Após a primeira etapa e com base na análise prévia dos achados, realizou-se a etapa qualitativa, por meio de cinco grupos focais com cinco equipes de Nasf-AB, representativas das macrorregiões, estabelecidas por sorteio e adequadas conforme viabilidade de acesso. Esta etapa contou com a participação de 43 profissionais, oriundos de cinco municípios, pertencentes a quatro das nove macrorregiões. Os grupos ocorreram entre setembro a novembro de 2017, conduzidos pelo pesquisador principal, com auxílio de um relator (realizava anotações sobre perfil e sequência dos falantes) e apoiadores (circulavam pela sala com os gravadores). Tiveram duração média de duas horas e os depoimentos que emergiram foram gravados e transcritos na íntegra.

A interpretação das informações ocorreu a partir da análise temática de conteúdo. ${ }^{10} \mathrm{Fez}-$ se o recorte do texto em unidades de registro, das quais emergiram duas categorias: planejamento do cuidado em saúde e práticas no uso de TICs. 
7 | Vendruscolo C, Geremia DS, Adamy EK, Vandresen L, Ferraz L

A pesquisa foi aprovada pelo Comitê de Ética em Pesquisa com Seres Humanos da UDESC sob parecer n⿳⺈ 1.812 .835 , de 08 de novembro de 2016, e respeitou todos os preceitos éticos e as diretrizes legislativas vigentes. Preservaram-se as identidades mediante codificação (letra M de “município”, seguida por número de ordem e letra P de “profissional”, seguida pelo número de ordem sequencial do grupo focal).

\section{Resultados}

Participaram da etapa quantitativa 359 profissionais que atuavam em 149 Nasf-AB no estado de SC, totalizando o alcance de 53,8\% dos municípios com Nasf-AB implantado. A tabela 1 apresenta as macrorregiões, o percentual por municípios e o total de profissionais que participaram da pesquisa.

Tabela 1- Cenário e participantes do estudo, Nasf-AB, Santa Catarina, 2018

\begin{tabular}{lccc}
\hline $\begin{array}{c}\text { Macrorregiões de Saúde de } \\
\text { SC }\end{array}$ & $\begin{array}{c}\text { Municípios com Nasf- } \\
\text { AB implantado }\end{array}$ & $\begin{array}{c}\text { Profissionais que } \\
\text { participaram da parte } \\
\text { quantitativa }\end{array}$ & $\begin{array}{c}\text { Profissionais que } \\
\text { participaram da parte } \\
\text { qualitativa }\end{array}$ \\
\hline 1. Grande Oeste & 75 & 94 & 26 \\
2. Meio Oeste & 43 & 38 & 0 \\
3. Nordeste & 9 & 25 & 0 \\
4. Grande Florianópolis & 39 & 54 & 9 \\
5. Foz do Rio Itajaí & 12 & 13 & 0 \\
6. Vale Itajaí & 40 & 49 & 4 \\
7. Serra Catarinense & 10 & 15 & 4 \\
8. Sul & 40 & 60 & 0 \\
9. Planalto Norte & 9 & 11 & 0 \\
Total & $\mathbf{2 7 7}$ & $\mathbf{3 5 9}$ & $\mathbf{4 3}$ \\
\hline
\end{tabular}

Dentre as profissões recomendadas pelo MS para a composição do Nasf-AB, 11 foram as que se destacaram, sendo mais frequentes: psicólogo $(n=96 / 27 \%)$, fisioterapeuta $(n=65 / 18,3 \%)$ e nutricionista $(n=63 / 17,7 \%)$; e menos frequentes: médico veterinário $(n=1 / 0,3 \%)$, terapeuta ocupacional $(n=2 / 0,6 \%)$ e sanitarista $(n=3 / 0,8 \%)$. A tabela 2 apresenta a frequência de respostas válidas para os diálogos dos profissionais do Nasf-AB com atores sociais da comunidade para busca de dados sobre o território. 
Utilização das tecnologias de informação e comunicação pelos núcleos ampliados de saúde... 8

Tabela 2- Apresentação da frequência de diálogos dos profissionais do Nasf-AB com atores sociais da comunidade, Santa Catarina, 2018

\begin{tabular}{lcc}
\hline Frequência de diálogos com atores sociais & $\mathrm{n}$ & $(\%)$ \\
\hline Diálogo com agentes comunitários de saúde & $\mathbf{3 5 7}$ & $(\mathbf{9 9 , 4 \% )}[\mathbf{1 0 0} \%]$ \\
Diariamente & 87 & $(24,4)$ \\
Até 3 vezes por semana & 91 & $(25,5)$ \\
Até 2 vezes por mês & 115 & $(32,2)$ \\
Raramente & 63 & $(17,6)$ \\
Nunca & 1 & $(0,3)$ \\
Diálogo com lideranças comunitárias & $\mathbf{3 5 8}$ & $(\mathbf{9 9 , 7})[\mathbf{1 0 0} \%]$ \\
1 dia por semana & 26 & $(7,3)$ \\
Até 2 vezes por mês & 60 & $(16,8)$ \\
Até 2 vezes por semestre & 46 & $(12,8)$ \\
Raramente & 179 & $(50,0)$ \\
Nunca & 47 & $(13,1)$ \\
Diálogo com conselheiros locais de saúde & $\mathbf{3 5 8}$ & $(\mathbf{9 9 , 7 \% )}[\mathbf{1 0 0} \%]$ \\
1 dia por semana & 22 & $(6,1)$ \\
Até 2 vezes por mês & 86 & $(24,0)$ \\
Até 2 vezes por semestre & 48 & $(13,4)$ \\
Raramente & 153 & $(42,7)$ \\
Nunca & 49 & $(13,7)$ \\
\hline
\end{tabular}

Entre os atores sociais, o agente comunitário de saúde (ACS) é o que dialoga com os profissionais do Nasf-AB, com uma frequência relativamente alta durante o mês de trabalho. As lideranças comunitárias e conselheiros locais de saúde têm uma frequência baixa de diálogo com os profissionais da equipe do Núcleo.

$\mathrm{Na}$ tabela 3, apresentam-se os registros como fonte de informação do Nasf-AB, considerando os sistemas de informação digital, prontuários físicos e mapa de território e, respectivamente, a frequência de uso.

Tabela 3- Apresentação do uso de registros da Unidade Básica de Saúde como fonte de informações para os profissionais do Nasf-AB, Santa Catarina, 2018

\begin{tabular}{lcc}
\hline Registros de informações e frequência de uso & $\mathrm{n}$ & $(\%)$ \\
\hline Sistemas de Informação em Saúde* & 357 & $(\mathbf{9 9 , 4 \% )}[\mathbf{1 0 0 \%}]$ \\
Diariamente & 248 & $(69,5)$ \\
Até 3 vezes por semana & 56 & $(15,7)$ \\
Até 2 vezes por mês & 28 & $(7,8)$ \\
Raramente & 14 & $(3,9)$ \\
Nunca & 11 & $(3,1)$ \\
Prontuário físico do usuário/família & $\mathbf{3 5 6}$ & $\mathbf{( 9 9 , 2 \% )}[\mathbf{1 0 0 \%}]$
\end{tabular}


9 | Vendruscolo C, Geremia DS, Adamy EK, Vandresen L, Ferraz L

\begin{tabular}{lcc}
\hline Registros de informações e frequência de uso & $\mathrm{n}$ & $(\%)$ \\
\hline Diariamente & 128 & $(36,0)$ \\
Até 3 vezes por semana & 54 & $(15,2)$ \\
Até 2 vezes por mês & 36 & $(10,1)$ \\
Raramente & 92 & $(25,8)$ \\
Nunca & 46 & $(12,9)$ \\
Mapa do território & $\mathbf{3 5 8}$ & $(\mathbf{9 9 , 7 \% )}[\mathbf{1 0 0} \%]$ \\
1 dia por semana & 25 & $(7,0)$ \\
Até 2 vezes por mês & 54 & $(15,1)$ \\
Até 2 vezes por semestre & 68 & $(19,0)$ \\
Raramente & 160 & $(44,7)$ \\
Nunca & 51 & $(14,2)$ \\
\hline
\end{tabular}

*Prontuário eletrônico.

Entre as fontes de informação que subsidiam as práticas dos profissionais, o prontuário eletrônico é utilizado em $87,1 \%$ na sua totalidade, considerando diariamente (36\%), até 3 vezes por semana $(15,2 \%)$, até 2 vezes por mês $(10,1 \%)$ e raramente $(25,8 \%)$. Entretanto, utiliza-se o mapa do território, 1 dia por semana $(7,0 \%)$, até 2 vezes por mês $(15,1 \%)$, até 2 vezes por semestre $(19,0 \%)$ e raramente com maior percentual $(44,7 \%)$, como fonte de informação.

Outro meio de obter informações utilizado na prática consiste no diálogo com profissionais das eSF são as conversas, que possibilitam a troca de informações, ocorrem de modo formal e informal, como observa-se na tabela 4

Tabela 4- Apresentação da frequência e meios de diálogos entre os profissionais do Nasf-AB e equipes da Saúde da Família, Santa Catarina, 2018

\begin{tabular}{lcc}
\hline Diálogos com as eSF & $\mathbf{N}$ & $\mathbf{( \% )}$ \\
\hline Reuniões agendadas/regulares com a equipe & $\mathbf{3 5 7}$ & $(\mathbf{9 9 , 4 \% )}[\mathbf{1 0 0} \%]$ \\
1 dia por semana & 159 & $(44,5)$ \\
Até 2 vezes por mês & 0 & $(0,0)$ \\
Até 2 vezes por semestre & 175 & $(49,0)$ \\
Raramente & 19 & $(5,3)$ \\
Nunca & 4 & $(1,1)$ \\
Conversas não-agendadas* com a equipe & $\mathbf{3 5 6}$ & $\mathbf{( 9 9 , 2 \% ) [ 1 0 0 \% ]}$ \\
Diariamente & 235 & $(66,0)$ \\
Até 3 vezes por semana & 72 & $(20,2)$ \\
Até 2 vezes por mês & 34 & $(9,6)$ \\
Raramente & 10 & $(2,8)$ \\
Nunca & 5 & $(1,4)$
\end{tabular}


Conversas virtuais ${ }^{* *}$ com profissionais da equipe

Diariamente

Até 3 vezes por semana

Até 2 vezes por mês

Raramente

Nunca
358

141

122

59

34

2 $(\mathbf{9 9 , 7 \% )}$ [100\%]

$(39,4)$

$(16,5)$

$(9,5)$

$(0,6)$

*corredores, copas, cozinhas, entre outros espaços da Unidade de saúde.

** Por e-mail ou telefônica.

São constantes os diálogos entre os profissionais do Nasf-AB e das eSF, ocorrendo durante as reuniões agendadas e de forma não resumida, em encontros nos espaços das unidades, por meios virtuais ou telefone.

$\mathrm{Na}$ análise dos resultados dos grupos focais, referente à categoria Planejamento do cuidado em saúde (representado pelas reuniões agendadas e uso de prontuário, formas de comunicação estruturadas), tais dispositivos de comunicação emergem nos depoimentos.

Hoje, a gente tem prontuário eletrônico nas Unidades de Saúde. Então, todas as ações do Nasf a gente evolui no prontuário eletrônico. (M4 P3)

O caso vem e já é submetido a uma ficha de acompanhamento do Nasf. Então, a equipe [eSF] passa para a gente o histórico, colocamos na ficha para acompanhar o que ocorreu nas visitas, imprimimos o que a gente evoluiu no prontuário eletrônico, anexamos à ficha e anotamos todas as datas das visitas. (M4 P4)

Ao prontuário do paciente, todos tem acesso. Então, dependendo da equipe da Estratégia, algumas costumam já fazer a evolução e colocar ali o que foi definido. Até as conversas informais vão para o prontuário eletrônico [...] (M4 P5)

A gente faz a reunião do Nasf, [...] se reúne semanalmente ou quinzenalmente. Então, a gente repassa casos nesses momentos, discute o que pode fazer, ações e planejamento. (M2 P4)

Como a gente está sempre na Unidade, [...] a reunião de equipe semanal está sempre disponível para que os nasfianos participem. Então, acabamos conversando. (M4 P2) 
11 | Vendruscolo C, Geremia DS, Adamy EK, Vandresen L, Ferraz L

Na segunda categoria, Práticas no uso de Tecnologias de Informação e Comunicação (representadas pelos meios informais de comunicação, com destaque para meios virtuais, de forma não cronológica e linear), os participantes enfatizaram que as trocas de informações também ocorrem em momentos informais, de encontros entre os profissionais, em diversos espaços das Unidades.

Discussão de casos e dados [estatísticos do território], algumas vezes é feito fora [da reunião de equipe], em conversas de corredor, [...] a comunicação ocorre em outros espaços. (M4 P1)

A gente trabalha bem parceria mesmo, senta e conversa. Aconteceu, chegou à situação, como a gente está na mesma unidade: fulana, eu tenho uma situação[...] (M1 P1)

Quando a gente consegue um momento nosso, a gente também conversa, discute as nossas prioridades no café da tarde. (M5 P1)

Importante destacar que casos reais e dados estatísticos do território são utilizados como fonte para a tomada de decisão. A comunicação por meios virtuais também é uma forma de repasse e troca de informações a respeito de pacientes e questões da comunidade:

Bastante conversação pelo telefone [...] todas as equipes recebem o cronograma mensal, nós estamos aqui hoje, mas lá na outra Unidade elas têm o cronograma e elas sabem que estamos aqui. Então, elas podem ligar diretamente ou a gente deixa nossos telefones para o gestor. (M5 P2)

A gente vai [na Unidade] para matriciar as equipes, eles mandam casos por Whats App e e-mail, como está a evolução do paciente, se piorou, se talvez precise da intervenção de algum profissional [...]. Conversamos com o enfermeiro por WhatsApp e e-mail, para conhecer mais dos indicadores, das características da região, para poder atuar. (M3 P1)

Costumamos utilizar o telessaúde para tirar dúvidas que não conseguimos resolver entre nós, conversando, ou às vezes, também, pesquisamos na internet [...]. (M1 P7) 
Utilização das tecnologias de informação e comunicação pelos núcleos ampliados de saúde... | 12

\section{Discussão}

A partir dos resultados, pode-se inferir que ainda existe uma lacuna para a incorporação e uso eficiente das tecnologias de informação e comunicação na APS, conforme encontrado na literatura. ${ }^{1-3,5}$ As equipes do Nasf-AB de Santa Catarina aplicam diversas formas de comunicação com as eSF para obter informações que auxiliem em suas práticas, mas é preciso refletir sobre os obstáculos para a estruturação, padronização e assimilação de tecnologias, a fim de enfrentálos.

Identificaram-se os principais interlocutores para a informação e comunicação, e os entraves, inovações e facilidades oriundos da inserção de tecnologias, com considerável impacto no processo de trabalho e nas formas de cuidado e gestão em saúde. O Nasf visa solucionar problemas pela comunicação, mediante habilidades singulares de cada membro da equipe. ${ }^{11}$ Coerente com essa diretriz, o estudo revela a comunicação como uma das principais ferramentas para o desenvolvimento de trabalho dos Nasf-AB. Nesse ínterim, o matriciamento pode se configurar como uma tecnologia central para um atendimento de acesso avançado e com resolutividade, inclusive, como espaço singular nas reuniões de equipe. ${ }^{8}$

Destaca-se a interlocução dos profissionais do Nasf com os ACS como fonte predominante de informação sobre a realidade e necessidades do território. Esse trabalhador ocupa lugar privilegiado na perspectiva da educação popular e representa importante fonte de informação na interface entre a comunidade e a equipe. Contudo, apesar do reconhecimento, estudos apontam que pouco se investe na qualificação e no seu processo crítico de comprometimento com a prática de liderança, embora se evidencie que a comunicação eficaz entre ACS, profissionais das equipes de Saúde da Família, Nasf e comunidade dá sentido e significado às práticas de saúde. ${ }^{12-14}$

Os dados quantitativos revelaram frequências baixas de diálogo com líderes comunitários e conselheiros locais de saúde, aspecto que não foi explorado nos grupos focais. Tal interação 
representa um desafio na implementação da diretriz participação social, prevista na legislação do SUS. Estudos ${ }^{12,14}$ advertem que o diálogo entre a comunidade e a eSF fortalece prerrogativas constitucionais do SUS, que se encontra em processo de mudanças e desacreditação. Formar alianças entre atores da comunidade e serviços de saúde é importante para ampliar o acesso e fortalecer a rede de atenção. ${ }^{15}$ Contudo, os conselheiros locais ainda têm uma invisibilidade para muitos profissionais de saúde, o que poderia ser resolvido com a divulgação e convite para as reuniões de equipe, estimulando a participação social, na comunidade e nos serviços de saúde. ${ }^{16}$

Em relação à utilização dos registros da Unidade, como o prontuário eletrônico, os dados quanti e qualitativos apontam para sua utilização de forma satisfatória, embora seja observada a necessidade de pensar novas formas de apropriação das informações, visando ao planejamento, acompanhamento e avaliação. Estudo que analisou o trabalho do Nasf-AB no território brasileiro, considerando sua integração com as eSF, em relação à utilização do prontuário comum entre as equipes, apresenta impacto positivo em $83 \%$ delas. ${ }^{17}$ Outra publicação sobre as percepções de gestores, profissionais e usuários, acerca do registro eletrônico, destaca sua importância no cuidado, questões envolvendo custos, sigilo, privacidade e implicações da centralização ou descentralização das informações. ${ }^{18} \mathrm{~A}$ utilização de registros eletrônicos na APS parece funcionar para a operacionalização do trabalho, como é possível verificar também pelos depoimentos dos profissionais neste estudo. No contexto brasileiro e mundial, de modo geral, a utilização de relatórios informatizados e registros informais é mais recorrente, permanecendo como desafio o reconhecimento dos potenciais do prontuário eletrônico ${ }^{1}$, o que difere da realidade encontrada no cenário em estudo.

Os mapas territoriais são importantes fontes de informação sobre a comunidade, mas são pouco utilizados pelos profissionais participantes deste estudo, de encontro a estudos nacionais que sinalizam a utilização desse tipo de ferramenta para a atuação das equipes na APS. ${ }^{1}$ Os mapas representam uma forma eficaz de visualizar informações sobre tópicos cruciais à atenção 
Utilização das tecnologias de informação e comunicação pelos núcleos ampliados de saúde... | 14

em saúde, uma vez que possibilitam a identificação de cuidados e visualização de padrões das doenças de uma população. Eles representam, portanto, uma modalidade de tecnologia que possibilita a melhoria da qualidade nos serviços de saúde. Entretanto, sua utilização pressupõe ações de territorialização atualizadas para problematizar, intervir, avaliar, monitorar e facilitar a comunicação, com troca de informações para o desenvolvimento do trabalho interdisciplinar e intersetorial, atendendo às demandas de cada território. ${ }^{19}$

As TICs podem ser meios para construir o conhecimento, mas não garantem sua geração. Os dados que delas se originam, em geral brutos, não geram estatísticas e informações que possam produzir evidências para a tomada de decisão. Porém, elas podem favorecer a agilidade e a disseminação da informação, que, associadas ao conhecimento, que é uma propriedade dos atores, pode apoiar as decisões colegiadas., ${ }^{5,20} \mathrm{Um}$ aspecto promissor desse levantamento tem a ver com o fato de os profissionais estarem utilizando as informações disponíveis - casos e dados estatísticos - para sua atuação em equipe.

Estudo revela que, no Brasil, não há tradição de diálogo personalizado entre diferentes profissionais, nem mesmo a construção coletiva de projetos terapêuticos nos serviços de saúde, o que se configura como um dos grandes desafios do SUS, considerando que a comunicação viabiliza e qualifica a atenção aos usuários, de forma pontual ou continuada. ${ }^{21}$ Nessa direção, também tem sido explorada na literatura a falta de atuação interprofissional na APS, justamente pela dificuldade de colaboração e interação entre os diversos núcleos de saberes e práticas. ${ }^{22}$ Todavia, os achados quanti e qualitativos desta pesquisa revelam conversas informais como fontes de informação para a atividade do $N a s f-A B$, e que as reuniões regulares de equipe figuram como espaço importante para o diálogo e a troca de informações e saberes entre os profissionais do Nasf e das eSF. Nesse sentido, vale lembrar que o modelo verticalizado de gestão/administração reflete o modelo biomédico, de encontro ao preconizado para o Nasf-AB. 
15 | Vendruscolo C, Geremia DS, Adamy EK, Vandresen L, Ferraz L

Assim, os profissionais do Nasf realizam o matriciamento, em certa medida, por meio das TICs. No Brasil, a ideia de matriciamento vincula-se à concepção teórica e metodológica que objetiva de dar suporte à cogestão de coletivos na APS, com funcionalidade a partir de três eixos de aplicação: apoio institucional, apoio matricial e clínica ampliada e compartilhada. O sucesso dessa proposta tem na colaboração elemento essencial, a partir de ações coletivas voltadas às necessidades do usuário, além do trabalho em equipe que contemple as perspectivas de cada profissional, o que significa lidar com conflitos. ${ }^{8,17,23}$ No contexto estudado, o matriciamento, ao encontro de outros estudos no estado, tangencia, como método aplicado, o trabalho das equipes do Nasf. ${ }^{23}$ Alguns depoimentos ainda revelam a utilização do telessaúde como tecnologia para o matriciamento. Coerente com outras pesquisas, os dados sinalizam a importância do aprendizado em serviço por meio da comunicação à distância. ${ }^{24}$

Com a evolução tecnológica, os métodos tradicionais de comunicação passam a ser substituídos por diferentes tecnologias, como estratégia de estabelecer a dialogicidade entre os integrantes da eSF e do Nasf e destes com a comunidade. Vislumbra-se que o espaço virtual passa a integrar um novo campo de comunicação. Entretanto, quando se discutem formas de comunicação entre pessoas e equipes, é imprescindível compreender que as TICs utilizadas devem ser ferramentas capazes de reduzir e eliminar discursos polifônicos ou os chamados ruídos, para que o processo seja claro e compreensível a todos os atores envolvidos, uma vez que as más interpretações podem gerar decisões equivocadas. Isto posto, há que se estabelecer estratégias de capacitação para que as informações sejam processadas, armazenadas e finalmente sirvam como meios de comunicação entre os profissionais de saúde e sejam ferramentas apoiadoras na operacionalização do cuidado assistencial, do matriciamento e da gestão dos serviços de saúde. 
Utilização das tecnologias de informação e comunicação pelos núcleos ampliados de saúde... | 16

\section{Conclusão}

No contexto estudado, a utilização das TICs pelas equipes dos Nasf-AB ainda representa um desafio, embora com alguns avanços para o cuidado na Atenção Primária. Elas contribuem para o fortalecimento do apoio matricial, como inovação e transformação nas relações de trabalho e, consequentemente, para um modelo assistencial integrador que poderá impactar nos cuidados primários. No entanto, os obstáculos identificados podem estar atrelados ao perfil dos profissionais que, por vezes, não demonstram competências necessárias ao uso da informação, bem como aos problemas estruturais para a inserção de novas tecnologias na APS.

Os dados oriundos dos sistemas de informações são utilizados para o planejamento das intervenções, com forte destaque para os prontuários eletrônicos. Os processos dialógicos durante as reuniões de equipe, e mesmo informais, entre os profissionais do Nasf-AB e das eSF, também são tecnologias que favorecem o cuidado, mediante o compartilhamento de informações e criação de possibilidades para resolver problemas. Percebe-se, no entanto, a necessidade de fortalecer a comunicação entre o Nasf- $A B$ e atores sociais, favorecendo a participação de lideranças comunitárias e conselheiros locais de saúde, e incorporar ao seu processo de trabalho outras tecnologias, como o mapa do território.

Embora a utilização das tecnologias ainda não atinja todo seu potencial para o cuidado no cenário estudado, a pesquisa poderá servir como estímulo para que gestores e equipes de saúde fortaleçam a utilização de ferramentas como o diálogo entre equipes, o mapa do território e a participação social, a fim de qualificar seu processo de trabalho.

No desenho deste estudo, orientado para a análise de uma estratégia de intervenção com características peculiares, foram selecionadas experiências aleatórias, limitando a generalização dos resultados. Sinaliza-se a diversidade dos municípios de SC, mas considera-se que foi possível refletir sobre o potencial das TICs utilizadas pelo Nasf para desenvolver o cuidado na 
17 | Vendruscolo C, Geremia DS, Adamy EK, Vandresen L, Ferraz L

APS, como também pode ser útil à definição de estratégias para a organização da rede de atenção à saúde.

Por fim, sugerem-se novos estudos, com ampliação de cenários e perspectiva longitudinal, para melhor compreender o fenômeno em toda sua complexidade e singularidade.

\section{Referências}

1. Santos AS, Fonseca Sobrinho D, Araújo LL, Procópio CSD, Lopes EAS, Lima AMLD, et al. Incorporação de tecnologias de informação e comunicação e qualidade na Atenção Básica em Saúde no Brasil. Cad Saúde Pública [Internet]. 2017 [acesso em 2019 nov 12];33(5):e00172815. Disponível em: https://www.scielosp.org/pdf/csp/2017.v33n5/e00172815/pt

2. Mota DN, Torres RAM, Guimarães JMX, Marinho MNASB, Araújo AF. Tecnologias da informação e comunicação: influências no trabalho da estratégia Saúde da Família. J Health Inform [Internet]. 2018 [acesso em 2019 nov 12];10(2):45-9. Disponível em: http://www.jhi-sbis.saude.ws/ojs-jhi/index.php/jhisbis/article/viewFile/563/330

3. Nogueira JWS, Rodrigues MCS. Comunicação efetiva no trabalho em equipe em saúde: desafio para a segurança do paciente. Cogitare Enferm [Internet]. 2015 [acesso em 2018 dez 20];20(3):636-40. Disponível em: http://www.saude.ufpr.br/portal/revistacogitare/wp-content/uploads/sites/28/2016/10/40016-162735-1PB.pdf

4. Lima JG, Giovanella L, Fausto MCR, Bousquat A, Silva EV. Atributos essenciais da Atenção Primária à Saúde: resultados nacionais do PMAQ-AB. Saúde Debate [Internet]. 2018 [acesso em 2019 ago 11];42(1):52-6. Disponível em: http://www.scielo.br/pdf/sdeb/v42nspe1/0103-1104-sdeb-42-spe01-0052.pdf

5. Pinheiro ALS, Andrade KTS, Silva DO, Zacharias FCM, Gomide MFS, Pinto IC. Gestão da saúde: o uso dos sistemas de informação e o compartilhamento de conhecimento para a tomada de decisão. Texto Contexto Enferm [Internet]. 2016 [acesso em 2019 jun 23];25(3):1-9. Disponível em: http://www.scielo.br/pdf/tce/v25n3/pt_0104-0707-tce-25-03-3440015.pdf

6. Ministério da Saúde (BR). Núcleo de Apoio à Saúde da Família - Volume 1: ferramentas para a gestão e para o trabalho cotidiano [Internet]. Brasília (DF): Ministério da Saúde; 2014 [acesso em 2018 jun 30]. Disponível em: http://bvsms.saude.gov.br/bvs/publicacoes/nucleo_apoio_saude_familia_cab39.pdf

7. Nascimento AG, Cordeiro JC. Núcleo ampliado de saúde da família e atenção básica: análise do processo de trabalho. Trab Educ Saúde [Internet]. 2019 [acesso em 2019 nov 12];17(2):e0019424. Disponível em: http://dx.doi.org/10.1590/1981-7746-sol00194 
Utilização das tecnologias de informação e comunicação pelos núcleos ampliados de saúde... $\mid 18$

8. Pagani R, Nascimento DDG. Equipe do Núcleo de Apoio à Saúde da Família: criar e recriar as possibilidades do seu fazer no cotidiano da saúde. In: Santos MLM, Kodjaoglanian VL, Ferrari FP, organizadoras. O NASF em cena: tecnologias e ferramentas de trabalho no cotidiano das equipes. Porto Alegre (RS): Rede Unida; 2016. 178 p.

9. Santos JLGS, Erdmann AL, Meirelles BHS, Lanzoni GMM, Cunha VP, Ross R. Integrating quantitative and qualitative data in mixed methods research. Texto Contexto Enferm [Internet]. 2017 [acesso em 2018 jun 30];26(3):e1590016. Disponível em: http://www.scielo.br/pdf/tce/v26n3/en_0104-0707-tce-26-03e1590016.pdf

10. Minayo MCS. O desafio do conhecimento: pesquisa qualitativa em saúde. $14^{a}$ ed. São Paulo (SP): Hucitec; 2014.

11. Ministério da Saúde (BR). Portaria no 2.436, de 21 de setembro de 2017. Aprova a Política Nacional de Atenção Básica, estabelecendo a revisão das diretrizes para a organização da Atenção Básica, no âmbito do Sistema Único de Saúde (SUS) [Internet]. Brasília (DF): Ministério da Saúde; 2017 [acesso em 2018 jun 30]. Disponível em: http://bvsms.saude.gov.br/bvs/saudelegis/gm/2017/prt2436_22_09_2017.html

12. Maciazeki-Gome RC, Souza CD, Baggio L, Wachs F. O trabalho do agente comunitário de saúde na perspectiva da educação popular em saúde: possibilidades e desafios. Ciênc Saúde Colet [Internet]. 2016 [acesso em 2019 jul 21];21(5):1637-46. Disponível em: http://www.scielo.br/pdf/csc/v21n5/1413-8123-csc-2105-1637.pdf

13. Torres GMC, Figueiredo IDT, Cândido JAB, et al. Comunicação terapêutica na interação profissional de saúde e hipertenso na estratégia saúde da família. Rev Gaúch Enferm [Internet]. 2017 [acesso em 2018 fev 26];38(4):e2016-0066. Disponível em: http://www.scielo.br/pdf/rgenf/v38n4/1983-1447-rgenf-38-04e2016-0066.pdf

14. Hartzler AL, Tuzzio L, Hsu C, Wagner EH. Roles and functions of community health workers in primary care. Ann Fam Med [Internet]. 2018 [acesso em 2019 ago 11];16(3):240-5. Disponível em: https://www.ncbi.nlm.nih.gov/pmc/articles/PMC5951253/

15. Oliveira DM, Deus NCP, Caçador BS, Silva EA, Garcia PPC, Jesus MCP, et al. Saberes e práticas de enfermeiros sobre a participação social na saúde. Rev Bras Enferm [Internet]. 2016 [acesso em 2019 ago 20];69(3):421-7. Disponível em: http://www.scielo.br/pdf/reben/v69n3/0034-7167-reben-69-03-0421.pdf

16. Hernández-Rincón EH, Lamus-Lemus F, Carratalá-Munuera C, Orozco-Beltrán D, Jaramillo-Hoyos CL, Robles-Hernández G. Building community capacity in leadership for primary health care in Colombia. MEDICC Rev [Internet]. 2017 [acesso em 2018 set 27];19:2-3. Disponível em: https://www.scielosp.org/pdf/medicc/2017.v19n2-3/65-70/en

17. Brocardo D, Andrade CLT, Fausto MCR, Lima SML. Núcleo de Apoio à Saúde da Família (Nasf): panorama nacional a partir de dados do PMAQ. Saúde Debate [Internet]. 2018 [acesso em 2019 ago 
22];42(1):130-44. Disponível em: http://www.scielo.br/pdf/sdeb/v42nspe1/0103-1104-sdeb-42-spe010130.pdf

18. Miwa MJ, Serapioni M, Ventura CAA. A presença invisível dos conselhos locais de saúde. Saúde Soc [Internet]. 2017 [acesso em 2019 jun 22];26(2):411-23. Disponível em: http://www.scielo.br/pdf/sausoc/v26n2/1984-0470-sausoc-26-02-00411.pdf

19. Costa JFR, Portela MC. Percepções de gestores, profissionais e usuários acerca do registro eletrônico de saúde e de aspectos facilitadores e barreiras para a sua implementação. Cad Saúde Pública [Internet]. 2018 [acesso em 2018 dez 02];34(1):e00187916. Disponível em: http://www.scielo.br/pdf/csp/v34n1/16784464-csp-34-01-e00187916.pdf

20. Landsberg GAP. e-Health and Primary Care in Brazil: concepts, correlations and trends. Rev Bras Med Fam Comunidade [Internet]. 2016 [acesso em 2019 ago 20];11(38):1-9. Disponível em: https://rbmfc.org.br/rbmfc/article/view/1234/827

21. Tesser CD. Núcleos de Apoio à Saúde da Família, seus potenciais e entraves: uma interpretação a partir da Atenção Primária à Saúde. Interface (Botucatu) [Internet]. 2017 [acesso em 2019 jul 21];21(62):565-78. Disponível em: http://www.scielo.br/pdf/icse/v21n62/1807-5762-icse-1807576220150939.pdf

22. Barros NF, Spadacio, Costa MV. Trabalho interprofissional e as práticas integrativas e complementares no contexto da Atenção Primária à Saúde: potenciais e desafios. Saúde Debate [Internet]. 2018 [acesso em 2019 maio 15];42(1):163-73. Disponível em: http://www.scielo.br/pdf/sdeb/v42nspe1/0103-1104-sdeb-42-spe01-0163.pdf

23. Vendruscolo C, Ferraz F, Tesser CD, Trindade LL. Núcleo ampliado de saúde da família: espaço de interseção entre atenção primária e secundária. Texto Contexto Enferm [Internet]. 2019 [acesso em 2019 set 21];28:e20170560. Disponível em: http://dx.doi.org/10.1590/1980-265X-TCE-2017-0560

24. Nilson LG, Maeyama MA, Dolny LL, Boin AF, Calvo MCM. Telessaúde: da implantação ao entendimento como tecnologia social. Rev Bras Tecnol Soc [Internet]. 2018 [acesso em 2019 nov 13]; 5(1):33-47. Disponível em: https://siaiap32.univali.br/seer/index.php/rbts/article/view/13400/7610

\section{Autor correspondente}

Carine Vendruscolo

E-mail: carine.vendruscolo@udesc.br

Endereço: Rua Mato Grosso 545 E, Jardim Itália, Chapecó/SC

CEP: 898014-080 
Utilização das tecnologias de informação e comunicação pelos núcleos ampliados de saúde... 20

\section{Contribuições de Autoria}

\section{1 - Carine Vendruscolo}

Contribuições: gerenciamento do projeto, coleta de dados, conceitualização, investigação, metodologia, redação inicial, revisão e edição, visualização.

\section{2 - Daniela Savi Geremia}

Contribuições: conceitualização, investigação, metodologia, redação inicial, revisão e edição, visualização.

\section{3 - Edlamar Kátia Adamy}

Contribuições: conceitualização, investigação, metodologia, redação inicial, revisão e edição, visualização.

\section{4 - Lara Vandresen}

Contribuições: gerenciamento do projeto, coleta de dados, conceitualização, investigação, metodologia, redação inicial, revisão e edição, visualização.

\section{5 - Lucimare Ferraz}

Contribuições: investigação, metodologia, revisão e edição, visualização.

\section{Como citar este artigo}

Vendruscolo C, Geremia DS, Adamy EK, Vandresen L, Ferraz L. Utilização das tecnologias de informação e comunicação pelos núcleos ampliados de saúde da família. Rev. Enferm. UFSM. 2019 [Acesso em: Anos Mês Dia];vol.9 e: 1-20. DOI:https://doi.org/10.5902/2179769239634 